\title{
MMIC amplifier-based receivers for Earth remote sensing
}

Douglas E. Dawson, Todd C. Gaier, Sander Weinreb, Matthew A. Morgan, Mary Wells

Douglas E. Dawson, Todd C. Gaier, Sander Weinreb, Matthew A. Morgan, Mary Wells, "MMIC amplifier-based receivers for Earth remote sensing," Proc. SPIE 5077, Passive Millimeter-Wave Imaging Technology VI and Radar Sensor Technology VII, (20 August 2003); doi: 10.1117/12.487381

SPIE. Event: AeroSense 2003, 2003, Orlando, Florida, United States 


\title{
MMIC Amplifier Based Receivers for Earth Remote Sensing
}

\author{
Douglas Dawson $^{a}$ and Todd Gaier ${ }^{a}$ and Sander Weinreb ${ }^{a}$ and Matt Morgan ${ }^{b}$ and Mary Wells ${ }^{a}$ \\ ${ }^{a}$ Jet Propulsion Laboratory, California Institute of Technology, Pasadena, CA \\ ${ }^{b}$ California Institute of Technology, Pasadena, CA
}

\begin{abstract}
We have developed amplifier based receivers using Indium Phosphide high electron mobility transistor (HEMT) monolithic microwave integrated circuit (MMIC) technology. These compact receivers are designed with atmospheric temperature and humidity sounding requirements in mind, operating at 100-125 GHz around the 118 GHz oxygen line, and at $160-185 \mathrm{GHz}$ near the $183 \mathrm{GHz}$ water line, with average noise temperatures of 1600 and $1200 \mathrm{~K}$ respectively. They are intended for applications where small volume and power consumption are critical. We will present laboratory data on the noise temperature of these receivers operated at room temperature and preliminary field data.
\end{abstract}

Keywords: MMIC, LNA, Radiometer

\section{INTRODUCTION}

Several chips and modules have been developed for compact $(<0.2 \mathrm{~kg})$, low power $(<0.4 \mathrm{~W})$, radiometric sensors operating in the 100-140 and 170-210 GHz ranges. These chips, developed utilizing InP transistor monolithic microwave integrated circuits (MMICs), have enabled a factor of 100 reduction in volume as compared to the sensors presently used in programs such as AMSU and MLS.

Millimeter-wave spectrometers have been used to monitor atmospheric temperature, pressure and humidity as well as upper atmospheric chemical composition. ${ }^{1,2}$ These are key data products for monitoring how the Earth system responds to natural and induced changes; particularly the effects of regional pollution on the global atmosphere, and the effects of global chemical changes on regional air quality. Current instruments, which use older millimeter-wave technologies, include UARS-MLS and AMSU-A\&B. Future versions of these missions will be revolutionized by reduced size, mass, cost and power required for MMIC technology at millimeter wavelengths. Atmospheric temperature, pressure and humidity can be measured by monitoring emission at frequencies on and near molecular lines (e.g. $\mathrm{O}_{2}$ at 59 or $118 \mathrm{GHz}$ and $\mathrm{H}_{2} \mathrm{O}$ at 22 or $183 \mathrm{GHz}$ ). Observation of $\mathrm{O}_{2}$ and $\mathrm{H}_{2} \mathrm{O}$ at higher frequencies results in much smaller antennas for a given resolution. In addition, the $100-140 \mathrm{GHz}$ range allows a measurement of carbon dioxide abundance from its $115 \mathrm{GHz}$ line, mid- and lower-tropospheric water vapor from continuum emission, and stratospheric ozone from several lines in this range. Additional data products in the 170-210 GHz band are, stratospheric ozone, and the abundance of $\mathrm{ClO}$ (the predominant form of reactive chlorine that destroys ozone), $\mathrm{N}_{2} \mathrm{O}, \mathrm{HNO}_{3}$, and volcanically-ejected $\mathrm{SO}_{2}$. Figure 1 below shows our modules (MATHS) compared to other earth science missions. A main theme of our work has been to develop and perform a laboratory demonstration of receivers suitable for an array instrument at these frequencies. These receivers also have a wide range of other applications where a compact, low-cost single radiometer for temperature and humidity is needed.

Our general goal was to improve the performance of our receivers over ones used in MLS. The MLS receiver utilizes the "gold brick" approach prevalent until approximately 1995, where each function of the receiver is realized in a machined block containing diode, transistor, or passive components. With MMIC technology each receiver function (or even multiple functions) is realized in a single monolithic chip of size of the order of a few $\mathrm{mm}$, which contains diode, transistor, and passive components. These MMIC chips are then connected with bond wires in a receiver module of size of a few $\mathrm{cm}$.

The rationale for MMIC technology, therefore, is a factor of 100 or more reduction in the volume of a millimeter wave receiver, coupled with low DC power and superior receiver performance. Table 1 below compares MLS to our performance goals.

Further author information: (Send correspondence to D.D)

D.D.: E-mail: douglas.e.dawson@jpl.nasa.gov, Telephone: 8183544395 


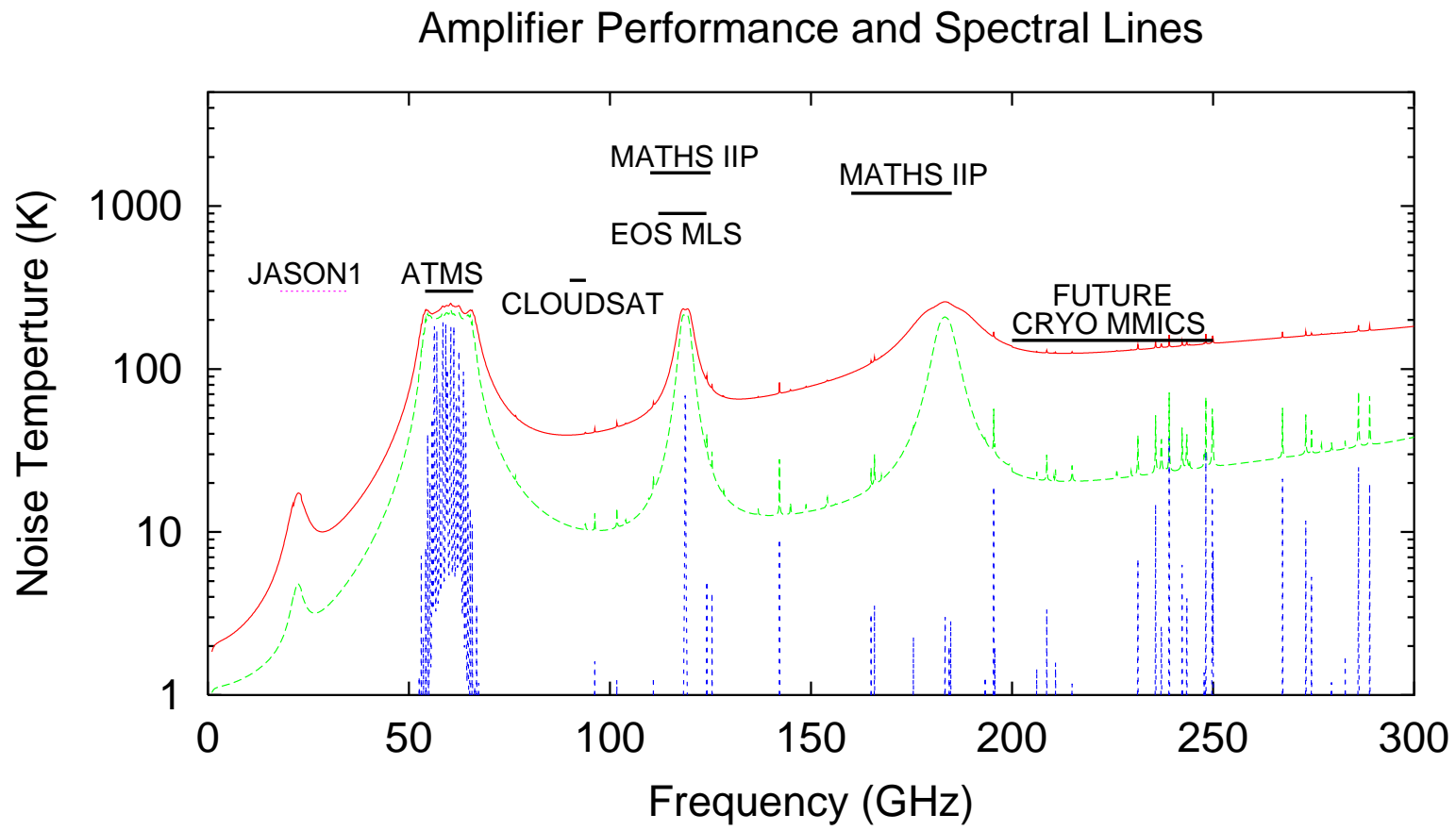

Figure 1. Noise Temperature of various amplifier enabled earth science missions and the zenith noise temperature of atmospheric chemical species at $0,3,30 \mathrm{~km}$

Table 1. Specifications of Developed Radiometers and Comparison with EOS/MLS 118 GHz Specifications

\begin{tabular}{|l|l|l|l|}
\hline Parameter & EOS/MLS & \multicolumn{2}{|c|}{ Design Goals } \\
\hline \hline RF Input Frequency Range, GHz & $115-123$ & $100-140$ & $170-210$ \\
\hline IF Output Frequency Range, GHz & $4-12$ & $10-50$ & $10-50$ \\
\hline Noise Figure/Temperature & $<7.4 \mathrm{~dB} / 1300 \mathrm{~K}$ & $<7.0 \mathrm{~dB} / 1160 \mathrm{~K}$ & $<8.0 \mathrm{~dB} / 1540 \mathrm{~K}$ \\
\hline RF/IF Gain & 15 & 30 & 30 \\
\hline Mass & $<2$ & $<0.2$ & $<0.2$ \\
\hline DC Power Requirement & $<5 \mathrm{~W}$ & $<0.3 \mathrm{~W}$ & $<0.3 \mathrm{~W}$ \\
\hline
\end{tabular}

\section{RECEIVER DESIGN}

\subsection{Module Design}

The basic module concept consists of gold plated brass blocks that have machined pockets for the MMIC chips. The input medium for the millimeter wave signal is standard WR8 or WR5 waveguide coming from the system antenna. The millimeter wave energy is translated from the waveguide mode to a planar field mode usable by the MMICs through a custom designed probe. ${ }^{3}$ This transformation is critical as any energy in a waveguide mode that enters the chip cavity area will not be amplified or downconverted by the MMICs. Not only is this energy lost to the system, but it can set up feedback mechanisms within the chip cavity, causing system oscillations or general receiver performance degradation. The low noise amplifier (LNA) chips are placed in custom designed cavities that help prevent the onset of these parasitic modes, and once the signal has been converted to the IF frequency band, less packaging care is needed.

The packaging concept calls for two separate blocks for each receiver. The first block houses at least two Proc. of SPIE Vol. $5077 \quad 43$ 
MMIC LNAs, and is more sensitive to module design. The second block contains up to one LNA followed by the rest of the downconverting chips. Figure 2 shows the overall packaging concept.

\section{OR 183 GHZ MMIC RECEIVER MODULE}

INTERIOR VIEW OF SPLIT BLOCK

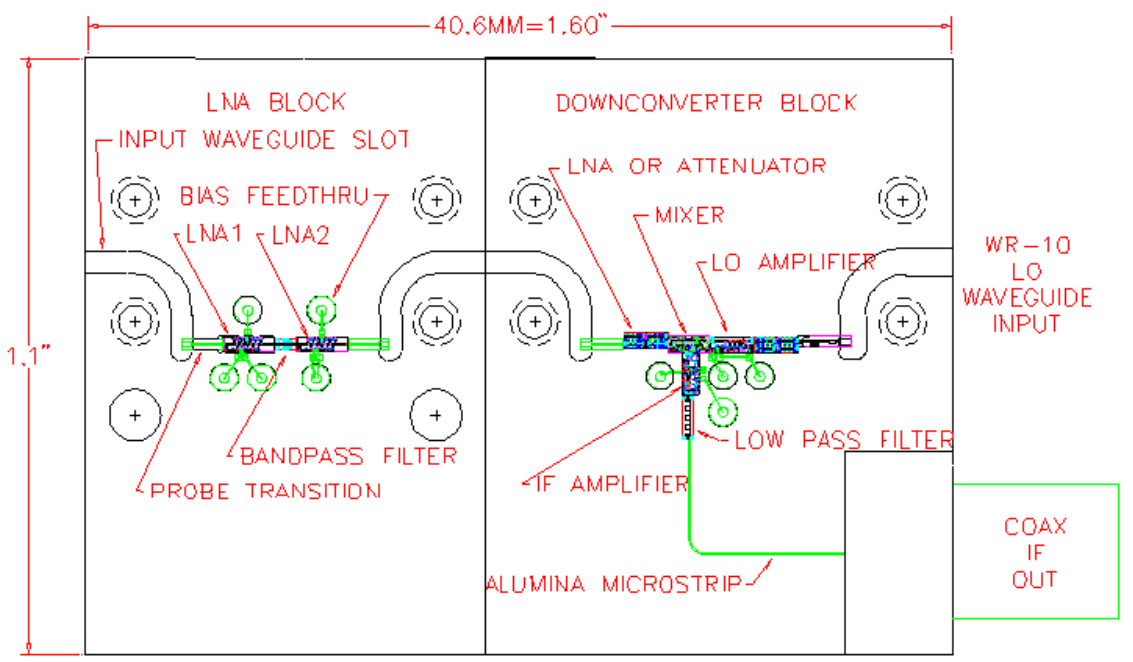

Figure 2. Receiver packaging concept

\subsection{0-140 GHz Module}

The 100-140 GHz receiver schematic is shown in Figure 3. A picture of the inside of part of the receiver assembly is shown in Figure 4. This receiver consists of 6 MMIC chips, all approximately $2 x 0.7 \mathrm{~mm}$ in size. The key to the dramatic size and weight reduction as compared to earlier systems is having all of the key components realized in MMIC technology, allowing close integration of these very small chips. Each block in the diagram

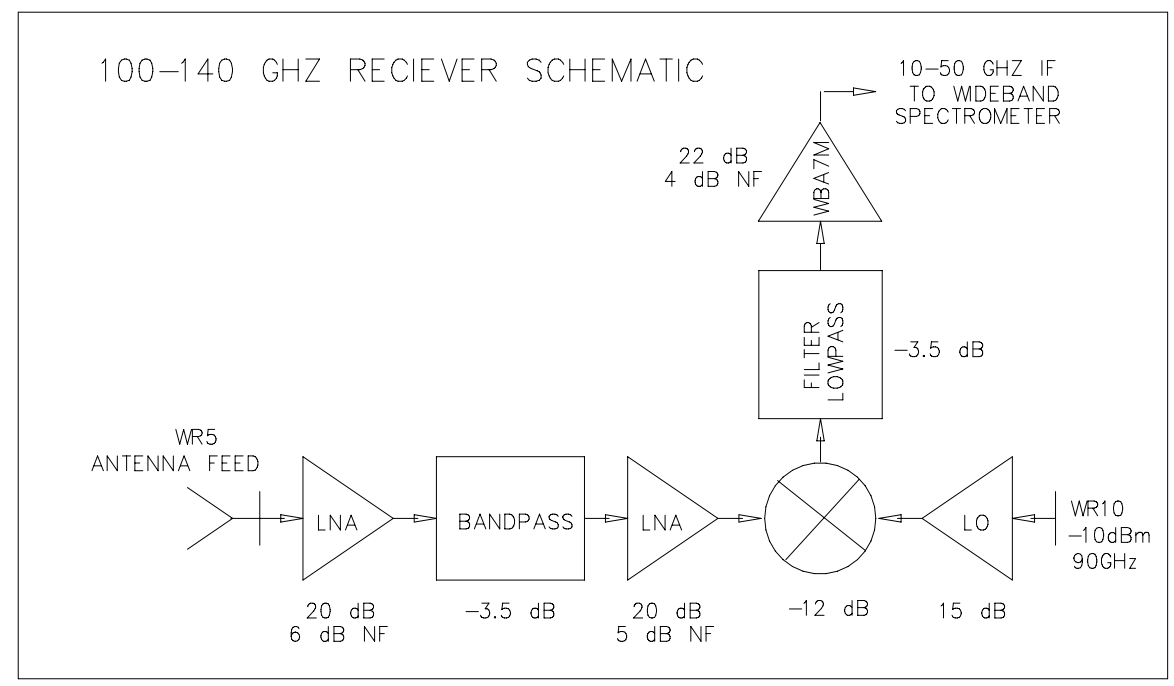

Figure 3. 100-140 GHz Module Schematic 


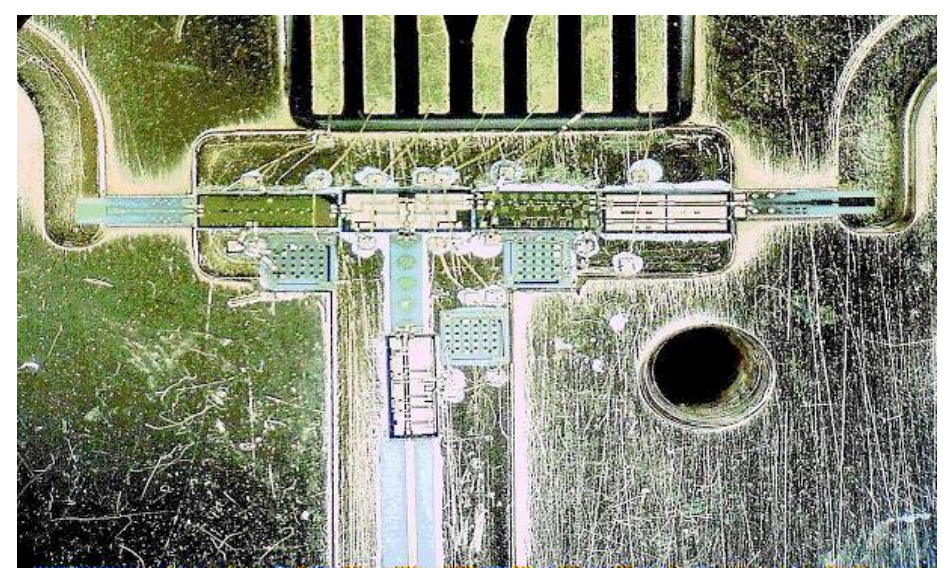

Figure 4. 100-140 GHz Module Picture of Downconverting Block

represents a MMIC or passive element, and each MMIC has key functionality in the receiver chain. The LNAs provides enough front end gain and low enough noise figure to provide good noise temperature over the band without large impacts from the elements later in the chain. The MMIC mixer has reasonably good conversion loss and is easier to integrate into the chain than a typical waveguide mixer assembly. The local oscillator (LO) amplifier provides enough output power $(+7 \mathrm{dBm})$ so that the LO distribution system outside of the receiver can be as low as $-10 \mathrm{dBm}$, thus significantly simplifying array systems that would employ many receivers. The intermediate frequency (IF) amplifier provides more than $20 \mathrm{~dB}$ of gain from 1-60 GHz, amplifying the broad IF band necessary to cover our 10-50 GHz IF goal.

\subsection{0-210 GHz Module}

The higher frequency module is schematically very similar to the $100-140 \mathrm{GHz}$ module. The input needs more care since the frequency is higher, and we used a 2nd harmonic mixer so that the LO frequency would be 90 $\mathrm{GHz}$, which is the same waveguide band as the $80 \mathrm{GHz} \mathrm{LO}$ for the lower frequency module. A schematic is shown below in Figure 5.

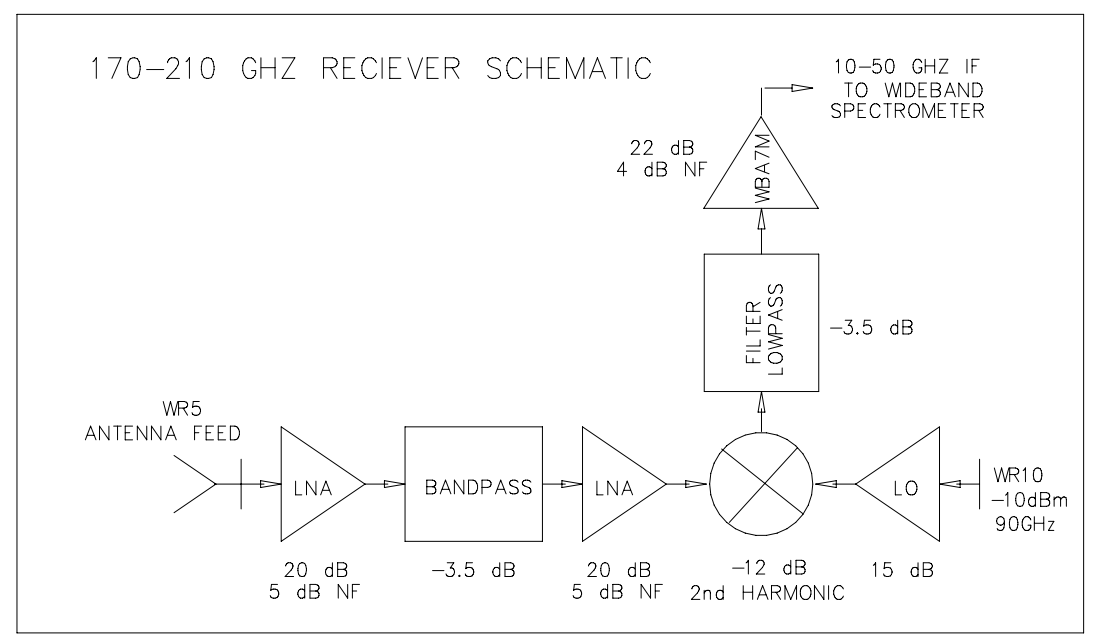

Figure 5. 170-210 GHz Module Schematic 


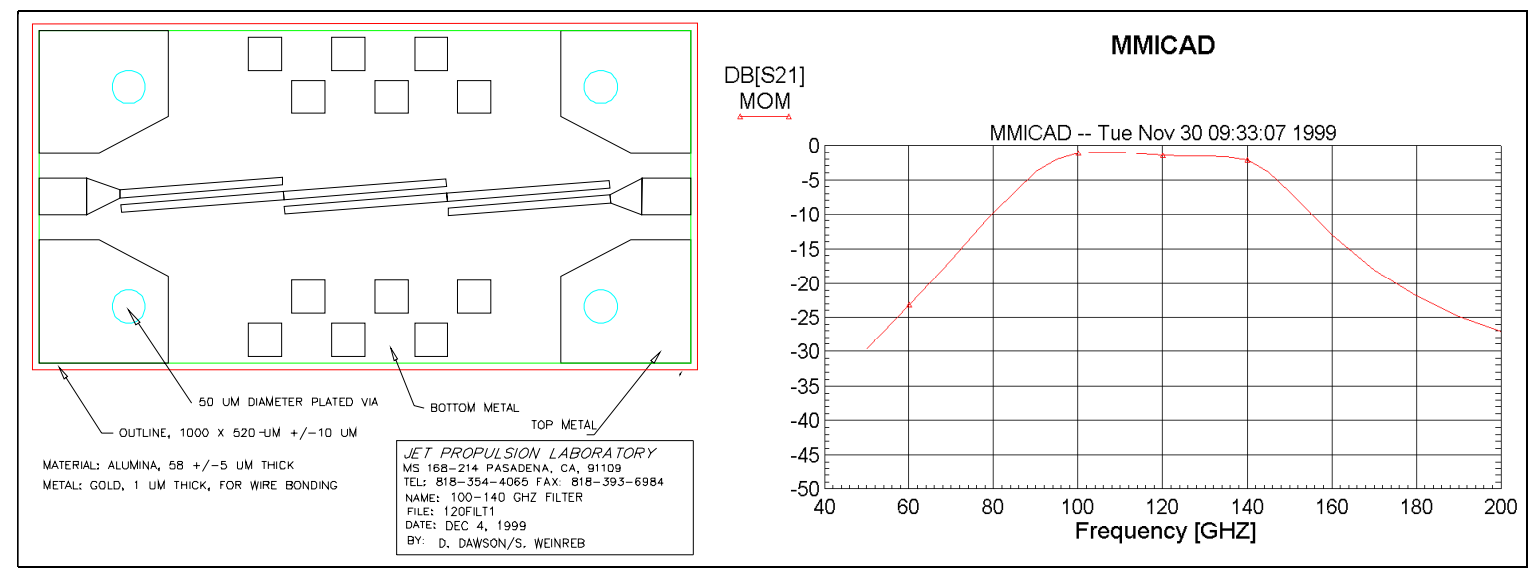

Figure 6. 100-140 GHz bandpass Filter

\subsection{Passive Structures}

In addition to the module design, several important passive circuits were also designed. The first is the probe that takes the waveguide input energy and forces it to a planar configuration for input to the MMIC chips, and the second is the RF bandpass filters in between the front end LNAs.

Three different probes were designed using the latest techniques from the literature. ${ }^{3}$ One probe was design for each input in WR5 and WR8, and one probe was designed for the WR10 LO input common to both receivers. The final longitudinal probe design was chosen over other more conventional designs to provide easy access for bonding to the MMICs, and also to provide one planar surface for mounting chips.

The passive filters provide rejection outside of the RF bandwidth of the receivers so that no unwanted energy is downconverted and detected. The filters were standard coupled line microstrip, electromagnetically simulated for optimum performance. The WR8 filter design is shown in Figure 6 along with its measured response.

\subsection{MMIC Design}

As stated in the module design section, each active element of a receiver was implemented in a MMIC chip, and a wide variety and several iterations were developed over the course of our program. Table 2 summarizes all of the MMIC chips.

The broad band IF amplifier is a state of the art LNA, with exceptionally wide bandwidth. The LO amplifier represent the first of the now standard higher power chips at $\mathrm{W}$ band frequencies. The mixer MMICs, developed by Matt Morgan, also represent the state of the art in MMIC technology. The final design for the $120 \mathrm{GHz}$ mixer is the first reported MMIC mixer in that band using amplifier gates as the mixing element, ${ }^{5}$ and the $180 \mathrm{GHz}$ mixer represents the only example of a second harmonic Schottky MMIC mixer known to the authors in that frequency range. By far the most challenging designs were the LNAs, and the best LNAs used in these modules represent the state of the art. ${ }^{6-8}$ The key to realizing these designs at such high frequency and bandwidths is a precise model for the HEMT in the frequencies of interest, and after several iterations we have very good LNAs in both bands.

The best 100-140GHz LNA is the 120LN8B, and the chip gain is shown in Figure 7. Two of these chips have been packaged together with a microstrip filter and optimized for ambient noise temperature. That package is the front end module described above for the WR8 receiver.

The $200 \mathrm{GHz}$ frequency range is the forefront of MMIC technology, and although most of our bandwidth requirements have been met, it is a difficult and very challenging range. The best WR5 LNA was developed independently by $\mathrm{TRW}^{4}$; and two of those chips and a filter have been packaged and tested for gain and noise at JPL, to become the WR5 front end module. 
Table 2. MMIC's Developed for the Receivers

\begin{tabular}{|c|c|c|c|}
\hline Function & Type & Foundry & Status \\
\hline \multicolumn{4}{|c|}{ 170-210 GHz Receiver } \\
\hline LNA & ALP114 & TRW & Tested, B (TRW design $\left.{ }^{4}\right)$ \\
\hline " & 200LN1 & TRW & Tested, B \\
\hline " & 200LN2 & HRL & Tested, B \\
\hline " & 200LN3 & HRL & Not Tested \\
\hline " & 200LN4 & HRL & Tested, D \\
\hline " & 190LN1 & Lockheed & Tested, C \\
\hline Mixer & 200MIX1 & UMS & Tested, A a \\
\hline IF Amplifier & WBA7 & HRL & Tested, A \\
\hline " & WBA7T & TRW & Tested, A a \\
\hline LO Amplifier & $70 \mathrm{LN} 3$ & HRL & Tested, A $\mathbf{a}$ \\
\hline \multicolumn{4}{|c|}{ 100-140 GHz Receiver } \\
\hline LNA & $120 \mathrm{LN} 1$ & TRW & Tested, B \\
\hline$"$ & 120LN1 & Lockheed & Tested, B \\
\hline " & $120 \mathrm{LN} 2$ & TRW & Tested, F Mask Error \\
\hline " & 120LN2 & TRW & Tested, F Fab Error \\
\hline$"$ & $120 \mathrm{LN} 3$ & TRW & Tested, C \\
\hline " & 120LN4 & HRL & Tested, D \\
\hline$"$ & 120LN5 & TRW & Tested, F \\
\hline " & $120 \mathrm{LN} 6$ & TRW & Tested, C \\
\hline 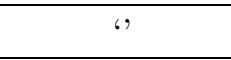 & 120LN7 & HRL & Not Tested \\
\hline " & 120LN8B & TRW & Tested, A \\
\hline " & $118 \mathrm{LN} 3$ & HRL & Tested, B \\
\hline Mixer & 120MIX1 & UMS & Tested, C \\
\hline$"$ & 120MIX2 & TRW & Tested, B \\
\hline IF Amplifier & WBA7 & HRL & Tested, A \\
\hline$"$ & WBA7T & TRW & Tested, A \\
\hline LO Amplifier & $70 \mathrm{LN} 3$ & HRL & Tested, A \\
\hline \multicolumn{4}{|c|}{$\begin{array}{c}\text { Table Key : A-Meets all Requirements, B-Needs Minor Improvement } \\
\text { C-Functional, D-Not Usable, F- Not Functional ; } \\
\text { a Denotes MMIC used in modules }\end{array}$} \\
\hline
\end{tabular}




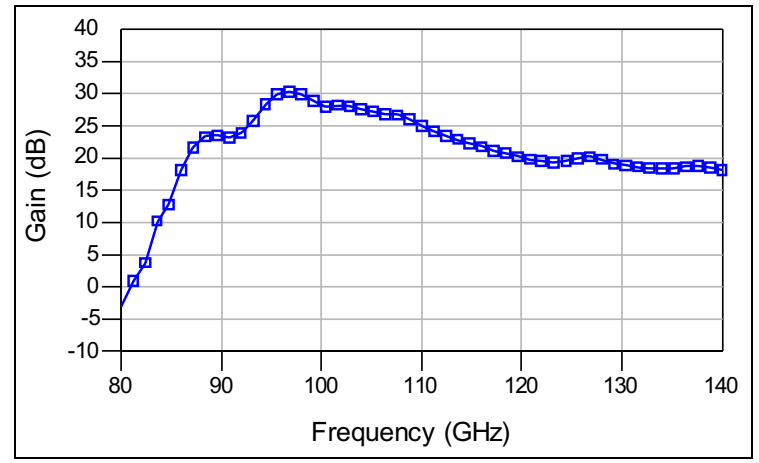

Figure 7. $120 \mathrm{GHz}$ LNA used in modules

\section{RECEIVER RESULTS}

The final assemblies for both the 100-140 GHZ and 170-210 GHZ bands were tested for receiver noise temperature at ambient temperature. The $170-210 \mathrm{GHz}$ module perfromed very well, although it did not vcover the entire RF bandwidth goal. The 100-140 GHz module had more problems, but had acceptable results. Table 3 compares the measured data to the program objectives.

Table 3. Results vs Objectives for Receiver Modules

\begin{tabular}{|c|c|c|}
\hline Parameter & Goal & Result \\
\hline \multicolumn{3}{|c|}{ 100-140 GHz Receiver } \\
\hline RF Input Frequency Range, GHz & $100-140$ & $110-125$ \\
\hline IF Output Frequency Range, $\mathrm{GHz}$ & $10-50$ & $20-35$ \\
\hline Noise Figure/Temperature & $<7.0 \mathrm{~dB} / 1160 \mathrm{~K}$ & $<8 \mathrm{~dB} / 1600 \mathrm{~K}$ \\
\hline RF IF Gain & 30 & $>20$ \\
\hline Mass, kg & $<0.2$ & 0.168 \\
\hline DC Power Requirement & $<0.3 \mathrm{~W}$ & $0.45 \mathrm{~W}$ \\
\hline \multicolumn{3}{|c|}{ 170-210 GHz Receiver } \\
\hline RF Input Frequency Range, GHz & $170-210$ & $160-185$ \\
\hline IF Output Frequency Range, $\mathrm{GHz}$ & $10-50$ & $0-25$ \\
\hline Noise Figure/Temperature & $<8.0 \mathrm{~dB} / 1540 \mathrm{~K}$ & $<7.0 \mathrm{~dB} / 1200 \mathrm{~K}$ \\
\hline RF IF Gain & 30 & $>30$ \\
\hline Mass, kg & $<0.2$ & 0.198 \\
\hline DC Power Requirement & $<0.3 \mathrm{~W}$ & $0.2 \mathrm{~W}$ \\
\hline
\end{tabular}

Figure8 shows the two receiver assemblies as they stood during the course of the program . By the end of the program the WR8 front end block was slightly larger, and the there were two similar blocks added to the WR5 front end. More work with these assemblies could shrink the overall volume even further.

\subsection{Detailed 100-140 GHz Receiver Results}

In general this receiver performed well, but it does have some packaging issues degrading the potential performance. The 120LN8B chip used as the front end LNA had $5 \mathrm{~dB}$ more gain per amplifier than predicted. This lead to an overall system gain that compressed the last LNA in the RF chain with the noise power generated by 


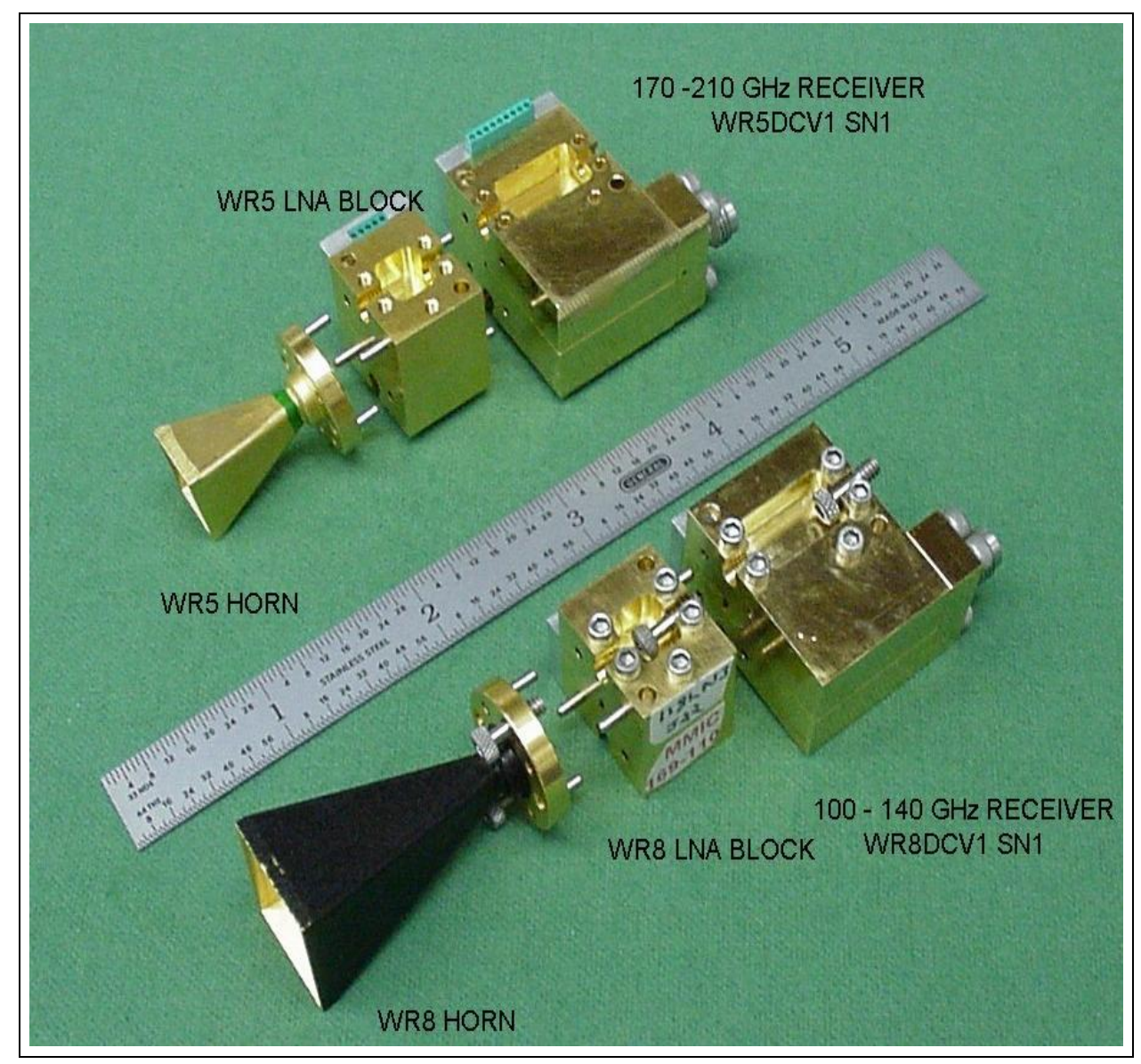

Figure 8. Assembled Modules

the first 2 LNAs. This compression has degraded the overall noise figure as measured by the Y factor method. Because of the higher gain per chip, a complete amplifier could be removed from the system and not significantly affect the system noise temperature. This would also lower the overall DC power consumption to less than $0.3 \mathrm{~W}$.

The 120LN8B also has a larger footprint than designed, and this caused some crowding of chips in the final package, forcing the waveguide probe to be off its optimum placement. The probe misalignment has caused an overall system input mismatch which shows up as a ripple pattern in the gain and noise figure.

Figure 9 shows the noise figure test set and the bias conditions of the receiver are listed below the figure. The $80 \mathrm{GHz}$ LO was supplied by a WR10 gunn oscillator, which is feed into a variable attenuator to set the LO level. That power goes into a coupler where the coupled port sends power to the LO input of the receiver, and the through port sends power to a power meter to calibrate the power level. The RF input is provided by absorber material used as a millimeter wave blackbody radiator. The temperature of the material is varied from room to liquid nitrogen temperatures, and this provides a know signal change that can be used to calculate the gain and noise figure of the receiver through the $\mathrm{Y}$ factor method. The IF signal is sent to an external mixer and then to a noise figure meter for the $\mathrm{Y}$ factor measurement, or to the spectrum analyzer for general system checks. 


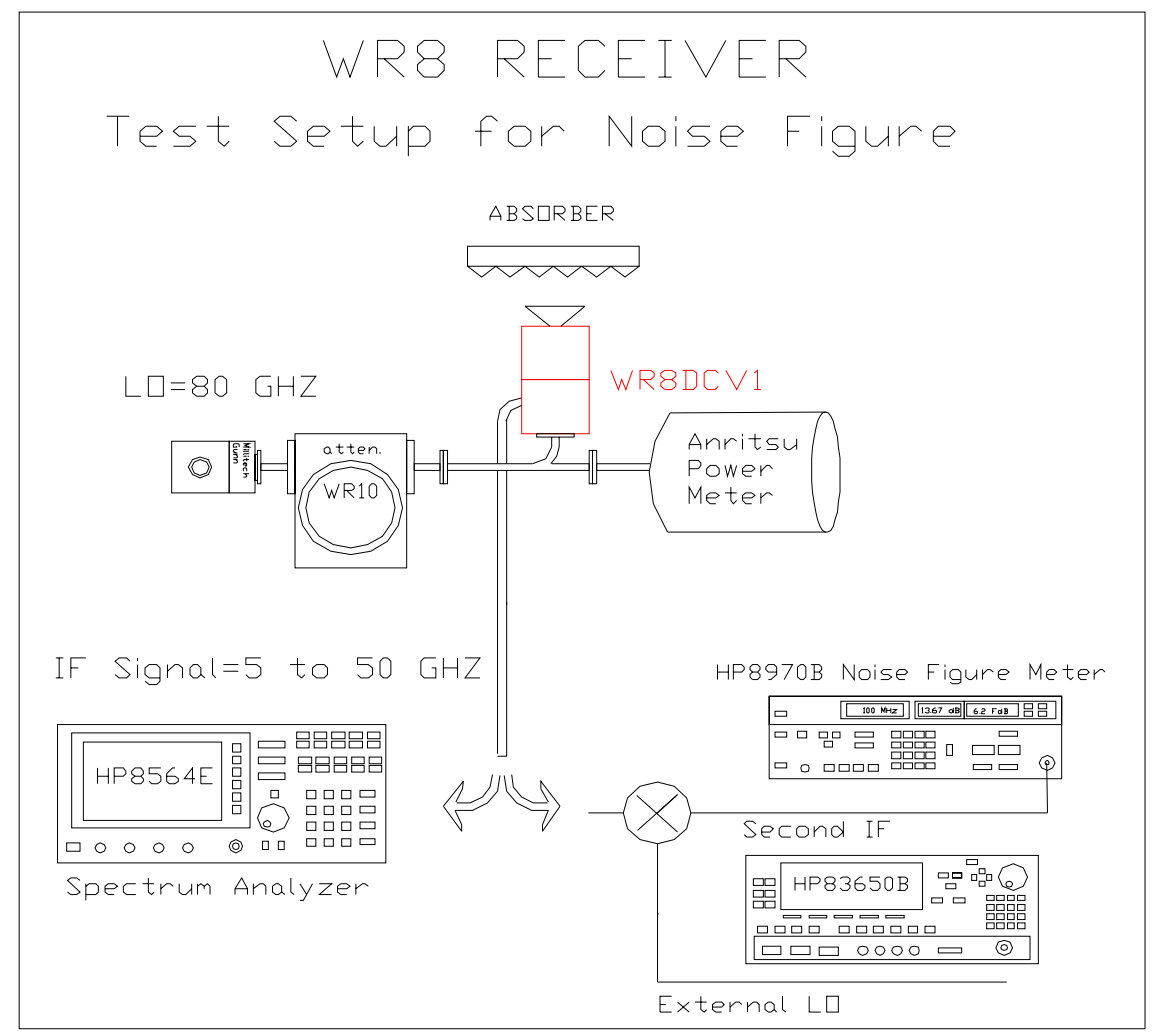

Figure 9. Noise and Gain Test Set

\section{Bias Conditions :}

- LNA $1: 1.0 \mathrm{~V} ; 39.98 \mathrm{~mA}$

- LNA $2: 1.5 \mathrm{~V} ; 59.28 \mathrm{~mA}$

- LNA $3: 2.484 ; 76.06 \mathrm{~mA}$

- Mixer : $3 \mathrm{~V} ; 1.744 \mathrm{~mA}$ ( w/LO applied)

- LO amp : $1.025 \mathrm{~V} ; 43.07 \mathrm{~mA}$

- IF amp $2.024 \mathrm{~V} ; 39.58 \mathrm{~mA}$

Using the test set as described, the noise was measured over the band and is shown in Figure 10. The data clearly shows the problems in the front end module. The compression has degraded the noise temperature from that of an individual amplifier, measured at $650 \mathrm{~K}$. The input mismatch shows as the ripple pattern in the data. Both these difficulties could be overcome with a slight redesign of the package and removal of a gain stage. Modules implementing these revisions have been made and will be flown on the HAMSR instrument as the 120 $\mathrm{GHz}$ channel. As a proof of concept measurement using this receiver as a atmospheric sensor, the temperature of the atmosphere was measured as a function of frequency. The measurement is done with the instrument at zenith, using a standard waveguide horn as an antenna. The noise of the receiver was calibrated during the measurement using the Y factor measurement, and the sky temperature plotted as function of frequency. No attempt was made to correct for the included angle of the horn. The results are in Figure 11. The artifact to the left of the oxygen peak is caused by the documented instrument compression. 


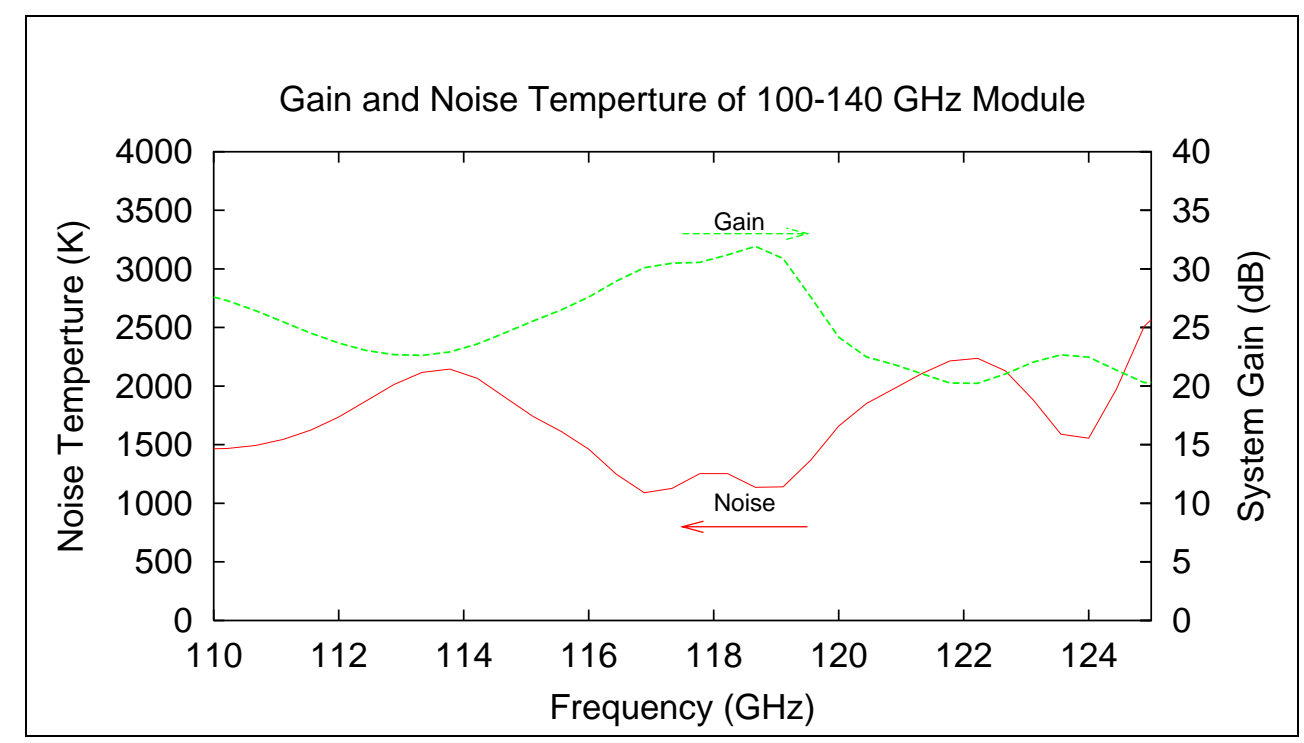

Figure 10. Noise Temperature and Gain for 100-140 GHz Module

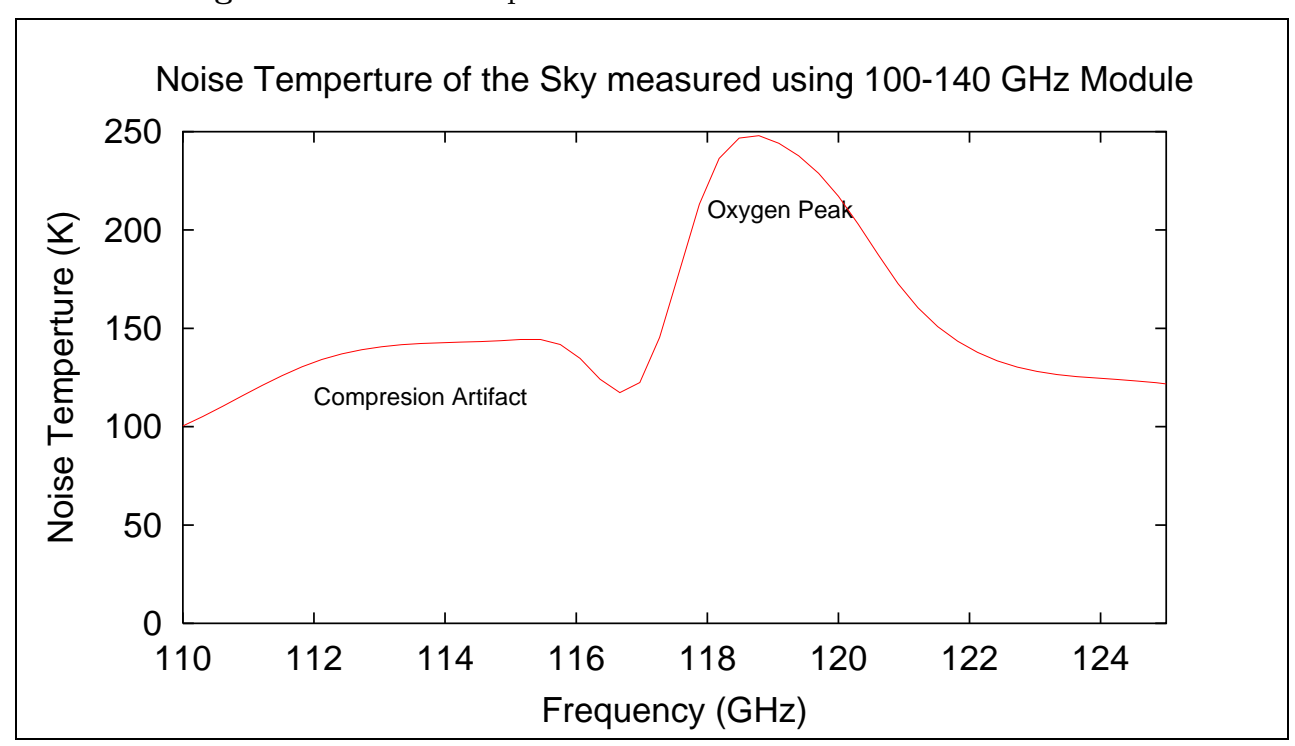

Figure 11. Sky Plot Using WR8 Receiver

\subsection{Detailed 170-210 GHZ RESULTS}

The WR5 module works very well. No compression was observed for the power levels of interest, and the noise figure is very flat. The module doesn't cover the entire desired band as the gain rolls off above $195 \mathrm{GHz}$, even thought the MMIC itself has gain to $210 \mathrm{GHz}$. This may be caused by the probe to MMIC interface, or unwanted modes in the RF cavity. Oscillatons were observed under some bias conditions, and chip redesign to minimize the MMIC footprint was completed and is in test and will allow for a better RF caivty design to mininmize the chance of oscillations.

The test set for room temperature is similar to the one used in the WR8 work, with the exception of the LO being supplied at $80 \mathrm{GHz}$ by a backward wave oscillator (BWO). Figure12 shows the room temperature receiver gain and noise temperature. 


\section{Bias Conditions :}

- LNA $1: 1.0 \mathrm{~V} ; 21.52 \mathrm{~mA}$

- LNA $2: 1.0 \mathrm{~V} ; 19.26 \mathrm{~mA}$

- LNA $3: 1.5 ; 22.5 \mathrm{~mA}$

- Mixer : 2V; $1.189 \mathrm{~mA}$ ( w/LO applied)

- LO amp : $1.0 \mathrm{~V} ; 41.05 \mathrm{~mA}$

- IF amp $2.0 \mathrm{~V} ; 38.50 \mathrm{~mA}$

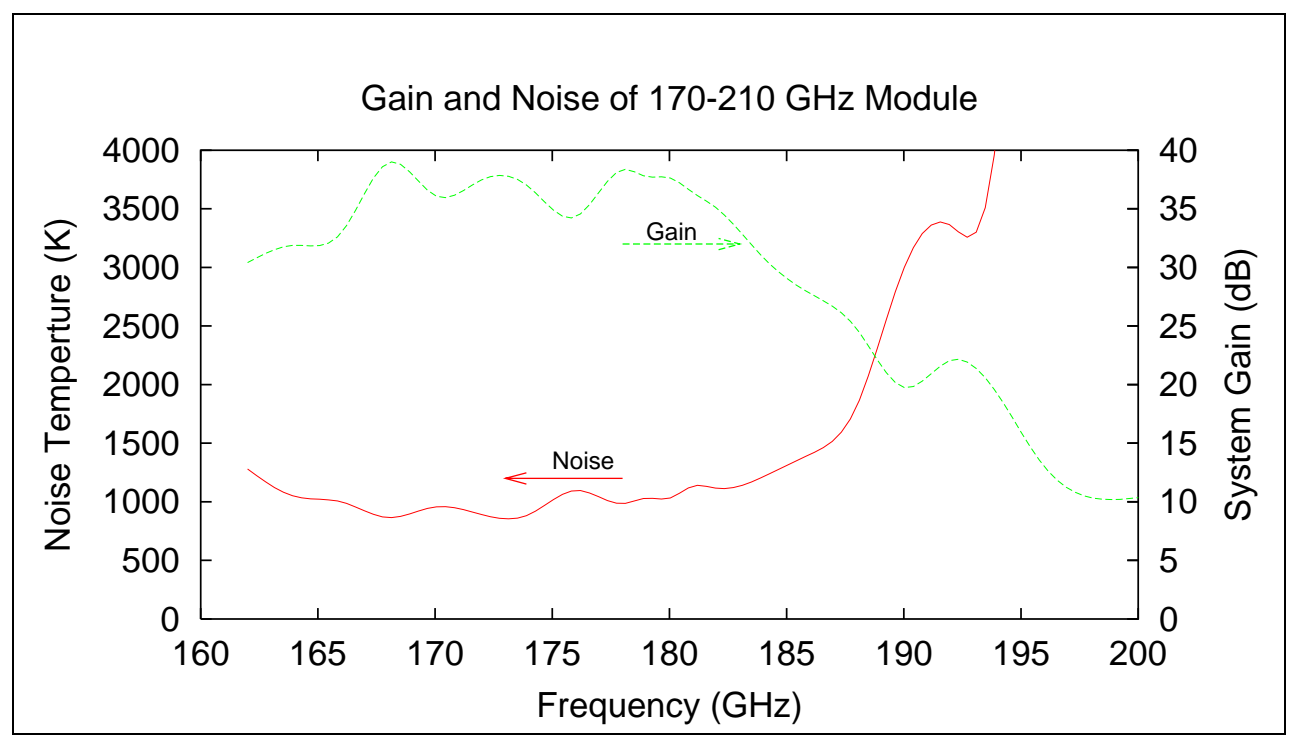

Figure 12. Noise Temperature and Gain for 170-210 GHz Module

\section{CONCLUSION}

We have developed amplifier based compact receivers designed with atmospheric temperature and humidity sounding requirements in mind, and where small volume and power consumption are critical. Both designs could be improved by some package redesign. The modules operate over 100-125 GHz around the $118 \mathrm{GHz}$ oxygen line, and at 160-185 GHz near the $183 \mathrm{GHz}$ water line with average noise temperatures of 1600 and $1200 \mathrm{~K}$ respectively.

\section{ACKNOWLEDGMENTS}

This work was performed at the Jet Propulsion Laboratory, California Institute of Technology, under a contract with the National Aeronautics and Space Administration. The authors would like to thank George Komar and Jonathan Hartley of the NASA ESTO office, and Loren Lemmerman of the JPL Earth Science and Technology Directorate for their support of this study. The authors also thank Mike Sholley, Matt Nishimoto, Ron Grundbacher, and Rohit Raja of TRW; and Mike Delaney and Adele Schmitz of HRL. Both HRL and TRW provided invaluable support for the development of the MMIC components. Also Gene Fisher for critical and precise assembly work. 


\section{REFERENCES}

1. M. Janssen, Atmospheric Remote Sensing by Microwave Radiometry, John Wiley, 1993.

2. J. Water, "Observations for chemistry (remote sensing): Microwave (ground, aircraft, balloon, satellite)," in Encyclopedia of Atmospheric Sciences, Academic Press, in press.

3. Y. Leong and S. Weinreb, "Full band waveguide-to-microstrip probe transitions," 1999 IEEE MTT-S Digest, pp. 1435-1438, 1999.

4. R. Raja, M. Nishimoto, B. Osgood, M. Barsky, M. Sholley, R. Quon, G. Barber, P. Liu, R. Lai, F. Hinte, G. Haviland, and B. Vacek, "A $183 \mathrm{GHz}$ low noise amplifier module with $5.5 \mathrm{~dB}$ noise figure for the ConicalScanning Microwave Imager Sounder (CMIS) program," in Microwave Symposium Digest, 2001 IEEE MTT-S 3, pp. 1955-1958, 2001.

5. M. Morgan and S. Weinreb, "A monolithic HEMT diode balanced mixer for 100-140 GHz," 1999 IEEE MTT-S International Symposium Digest, pp. 99-102, 2001.

6. P. Smith, "Status of InP HEMT technology for microwave receiver applications," IEEE MTT 44, pp. 23282333, December 1996.

7. H. Wang, R. Lai, Y. Kok, T. Huang, M. Aust, Y. Chena, P. Siegel, T. Gaier, R. Dengler, and B. Allen, "A 155-GHz monolithic low-noise amplifier," IEEE MTT 46, pp. 1660-1660, November 1998.

8. S. Weinreb, T. Gaier, M. Barsky, Y. Leong, and L. Samoska, "High-gain 150-215GHz MMIC amplifier with integral waveguide transitions," IEEE Microwave and Guided Wave Letters 9, July 1999. 